\title{
Müssen bei der Behandlung auf See Infektionen durch MRSA-Stämme berücksichtigt werden?
}

\author{
Lucas R, Boniface K, Roberts K, Kane E. Suspected methicillin-resistant Staphylococcus aureus infections at sea. \\ Int Marit Health 2007; 58: 1-4
}

Thema: Methicillinresistente Staphylococcus-aureus-Stämme (MRSA) sind als Erreger von Krankenhausinfektionen weltweit verbreitet. Seit einigen Jahren werden zunehmend MRSA-Infektionen bei Personen ohne erkennbare Risikofaktoren festgestellt. Diese sogenannten „community acquired“ MRSA (CA-MRSA) führen in über $90 \%$ zu eitrigen Haut- und Weichteilinfektionen. Hauterkrankungen gehören neben Unfällen und respiratorischen Erkrankungen zu den häufigsten Behandlungsanlässen an Bord. Wie häufig CA-MRSA die Ursache von Infektionen bei Seeleuten sind ist nicht bekannt, da eine mikrobiologische Diagnostik bei der Behandlung auf Frachtschiffen praktisch unmöglich ist. Durch die telemedizinischen Beratungsstellen haben Seeleute Zugang zu ärztlicher Beratung für die Behandlung an Bord. Die Statistiken der telemedizinischen Beratungszentren liefern wichtige Informationen zur Morbidität und Mortalität von Seeleuten.

Projekt: Die Autoren der hier vorgestellten Studie betreuen die telemedizinische Beratungsstelle an der George Washington Universität. Die Kommunikation zwischen dem behandelnden Seemann an Bord und dem beratenden Arzt läuft über Satellitentelefon oder E-Mail, nicht immer können digitale Bilder übertragen werden. Lucas et al. untersuchten anhand ihrer Beratungsprotokolle und Bilder retrospektiv die Inzidenz von Haut- und Weichteilinfektionen bei Seeleuten für die Jahre 2002-2006. Kriterien für die Klassifikation „verdächtig für MRSA“ waren der Nachweis von Eiter, kleinen Abszessen oder Furunkeln sowie Spinnenbissen, aber auch die Notwendigkeit der Inzision/Drainage.

Ergebnis: Im Jahr 2002 wurden 200, im Jahr 2006520 telemedizinische Beratungen durchgeführt. Der Anteil von Hautinfektionen unter allen Beratungsfällen stieg von 5,6\% im Jahr 2002 auf 8,8\% im Jahr 2006 an. Bei etwa 25\% aller Haut- und Weichteilinfektionen in dem 5-JahresZeitraum war eine Inzision und Drainage durch den Schiffsoffizier oder einen Arzt im Hafen notwendig. Die Autoren schätzten auf der Grundlage der klinischen Charakteristika, dass im Jahr 2002 36\% und im Jahr 2006 74\% aller Hautbefunde verdächtig für eine Infektion durch MRSA waren. Fazit: Im 5-Jahres-Untersuchungszeitraum der Studie der telemedizinischen Beratungsstelle der George Washington Universität nahm die Inzidenz von Haut- und Weichteilinfektionen bei Seeleuten deut-

lich zu und der Anteil der auf MRSA verdächtigen Befunde war auf mehr als das Doppelte gestiegen. Aus Sicht der Autoren müssen die Seeleute mit der chirurgischen Behandlung von Abszessen vertraut sein. Weiterhin weisen sie auf die Notwendigkeit einer Bevorratung von Antibiotika hin, die auch eine mögliche MRSA-Infektion abdecken und betonen die Bedeutung der allgemeinen Hygiene an Bord.

Dr. Clara Schlaich, Hamburg

\section{Kommentar}

Die Besonderheiten des Arbeitsplatzes auf See führen es mit sich, dass Seeleute auch heute noch berufsspezifischen gesundheitlichen Risiken wie Unfällen, erhöhter UV-Strahlung oder psychomentalen Belastungen ausgesetzt sind. Aus infektionshygienischer Sicht ist auch das enge, einem Haushaltskontakt vergleichbare, oft monatelange Zusammenleben von Seeleuten aus unterschiedlichen Weltregionen ein Risikofaktor für die Übertragung von ansteckenden Erkrankungen.

Die "Centers for Disease Control and Prevention“ in den USA benennen die „five C's of MRSA transmission“ mit: „crowding“, „frequent skin-to-skin contact“, „compromised skin integrity“, "contaminated items“ und "surfaces and lack of cleanliness“ [1]. La Mar et al. beschreiben das Auftreten einer Hautinfektion durch MRSA bei 2 Rekruten an Bord eines Marineschiffes. Wie die Nasenabstriche der 125 Mannschaftskollegen im selben Wohnbereich ergaben, waren $6,4 \%$ asymptomatische MRSA-Träger [2].

Der Untersuchung von Lucas et al. zufolge sind Hautinfektionen durch MRSA bei Seeleuten möglicherweise häufiger, als bisher angenommen. Wahrscheinlich wurde die Häufigkeit von MRSA verdächtigen Befunden durch Fehlklassifikationen überschätzt. Genauere Daten können nur Studien mit mikrobiologischer Probenentnahme liefern. Deren Ergebnisse werden direkte Auswirkungen für die medizinische Behandlung der Seeleute haben und darüber hinaus zum Verständnis der globalen Epidemiologie von CA-MRSA beitragen.

Die Prävalenz der bisher bekannten CA-MRSA Stämme unterscheidet sich geografisch erheblich. Die Bedeutung des Reiseverkehrs und von Migrationsbewegungen bei der globalen Ausbreitung verschiedener CA-MRSA-Stämme sind Gegenstand von Untersuchungen. CAMRSA-Stämme wurden praktisch in allen industrialisierten Ländern nachgewiesen [1, 3]. In Asien und Osteuropa, wo ein Großteil der Seeleute der Handelsflotte rekrutiert wird, ist die Epidemiologie von CA-MRSA bis auf wenige Fallberichte nicht bekannt [4].

Ärzte, die in den Häfen eine chirurgische Behandlung von Panaritien, Furunkeln/Karbunkeln oder Abszessen des Haut-Weichteilgewebes bei Seeleuten durchführten, sollten die Entnahme eines Abstriches aus dem Eiter und eine mikrobiologische Untersuchung erwägen und in der Auswahl des Antibiotikums eine mögliche MRSA-Infektion berücksichtigen. Die Hafenärztlichen Dienste können die behandelnden Ärzte bei der Kommunikation der Befunde an die Reedereien unterstützen.

Dr. Clara Schlaich, Hamburg

\section{Literatur}

1 DeLeo FR, Otto M, Kreiswirth BN, Chambers HF. Community-Associated meticillin-resistant Staphylococcus aureus. Lancet 2010; 375: 1557-1567

2 LaMar JE, Carr RB, Zindermann C, McDonald K. Sentinel cases of community-acquired methicillin-resistant Stapylococcus aureus onboard a naval ship. Mil Med 2003; 168: 135-138

3 Otter JA, French GL. Molecular epidemiology of community-asscosiated meticillin-resistant Staphylococcus aureus in Europe. The Lancet Infect Dis 2010; 10: 227-239

4 Nickerson EK, West TE, Nicholas PD, Peacock SJ. Staphylococcus aureus disease and drug resistance in resource-limited countries in south and east Asia. Lancet Infect Dis 2009; 9: 130-135 